\title{
The Effect of Cesarean Delivery Skin Incision Approach in Morbidly Obese Women on the Rate of Classical Hysterotomy
}

\author{
Brian E. Brocato, ${ }^{1}$ Edwin M. Thorpe Jr., ${ }^{1}$ Luis M. Gomez, \\ Jim Y. Wan, ${ }^{2}$ and Giancarlo Mari ${ }^{1}$ \\ ${ }^{1}$ Department of Obstetrics and Gynecology, University of Tennessee Health Science Center, 853 Jefferson Avenue Suite E102, \\ Memphis, TN 38163, USA \\ ${ }^{2}$ Department of Preventive Medicine, University of Tennessee Health Science Center, 853 Jefferson Avenue Suite E102, \\ Memphis, TN 38163, USA
}

Correspondence should be addressed to Brian E. Brocato; bbrocato@uthsc.edu

Received 15 August 2013; Accepted 22 October 2013

Academic Editor: Roberta B. Ness

Copyright (c) 2013 Brian E. Brocato et al. This is an open access article distributed under the Creative Commons Attribution License, which permits unrestricted use, distribution, and reproduction in any medium, provided the original work is properly cited.

Objective. To assess the risk of classical hysterotomy and surgical morbidity among women with a body mass index (BMI) greater than $40 \mathrm{~kg} / \mathrm{m}^{2}$ who underwent a supraumbilical incision at the time of cesarean delivery. Methods. We conducted a retrospective cohort study in women having a BMI greater than $40 \mathrm{~kg} / \mathrm{m}^{2}$ who underwent a cesarean delivery of a live, singleton pregnancy from 2007 to 2011 at a single tertiary care institution. Intraoperative and postoperative outcomes were compared between patients undergoing supraumbilical vertical (cohort, $n=45$ ) or Pfannenstiel (controls, $n=90$ ) skin incisions. Results. Women undergoing supraumbilical incisions had a higher risk of classical hysterotomy (OR, 24.6; 95\% CI, 9.0-66.8), surgical drain placement (OR, 6.5; 95\% CI, 2.6-16.2), estimated blood loss greater than 1 liter (OR, 3.4; 95\% CI, 1.4-8.4), and longer operative time (97 \pm 38 minutes versus $68 \pm 30$ minutes; $P<.001)$ when compared to subjects with Pfannenstiel incisions (controls). There was no difference in the risk of wound complication between women undergoing supraumbilical or Pfannenstiel incisions (OR, 2.7; 95\% CI, 0.9-8.0). Conclusion. In women with a BMI above $40 \mathrm{~kg} / \mathrm{m}^{2}$, supraumbilical incision at the time of cesarean delivery is associated with a greater risk of classical hysterotomy and operative morbidity.

\section{Introduction}

Obesity is a common, serious health condition that poses major health risks to individuals and increases costs to society. It is estimated that 97 million adults in the United States are obese as defined as body mass index 30 or greater and approximately one-third of childbearing-age women in the United States are obese [1]. Obesity places pregnant women and their offspring at increased risk for complications such as hypertensive disorders, diabetes, cesarean delivery, macrosomia, and stillbirth [2-7].

As is true for any abdominal surgery, cesarean delivery presents clear challenges in obese women. In pelvic surgery, low transverse or Pfannenstiel skin incisions give adequate exposure while providing a good cosmetic result [8]. Still, the pannus may limit intraoperative exposure when using a Pfannenstiel incision; in that case a vertical incision is generally preferred. To avoid the dissection of thickened layers of adipose tissue below the umbilicus, a supraumbilical vertical incision arises as an attractive alternative approach. However, this type of incision (supraumbilical) is often associated with limited intraoperative exposure, which increases operative difficulty and surgical morbidity. There is a paucity of information regarding the risks associated with supraumbilical vertical incision at the time of cesarean. Consequently, we sought to investigate whether supraumbilical vertical cesarean incisions in women with a body mass index greater than $40 \mathrm{~kg} / \mathrm{m}^{2}$ are associated with classical hysterotomy as the result of restricted intraoperative exposure and other postoperative morbidities.

\section{Materials and Methods}

Hospital records of all pregnant women with a body mass index greater than $40 \mathrm{~kg} / \mathrm{m}^{2}$ who underwent cesarean 
TABle 1: Demographics.

\begin{tabular}{lccc}
\hline Characteristics & Supraumbilical & Pfannenstiel & $N=90$ \\
\hline Age & $N=43$ & $26 \pm 6$ & $<$ value \\
Average body mass index & $32 \pm 5$ & $56 \pm 6$ & $<.0001$ \\
Gestation at delivery & $64 \pm 10$ & $38.3 \pm 3$ & .0001 \\
African American & $36.5 \pm 2.9$ & $77(86 \%)$ & .0015 \\
Diabetes & $37(86 \%)$ & $8(9 \%)$ & .6655 \\
Hypertension & $21(49 \%)$ & $39(43 \%)$ & .2722 \\
Preeclampsia & $23(53 \%)$ & $31(34 \%)$ & .2780 \\
Tobacco use & $19(44 \%)$ & $10(11 \%)$ & 1.0000 \\
Previous abdominal surgery & $4(9 \%)$ & $44(49 \%)$ & .9956 \\
\hline
\end{tabular}

delivery from January 2007 to January 2011 were reviewed after approval from The University of Tennessee Health Science Center Institutional Review Board. Both dictated and written operative reports were used to identify the types of uterine and skin incisions. Our cohort included all patients who underwent supraumbilical vertical skin incisions. Patients who underwent Pfannenstiel incisions comprised controls. The control participants were selected chronologically; cohort and controls were matched at a $1: 2$ ratio.

Our primary outcome was the occurrence of classical hysterotomy. Assuming a prevalence of the primary outcome of $5 \%$ in the control group and considering a power of $80 \%$ and 2 -sided alpha $=0.05$, we estimated that a sample size of 34 subjects in each group was required for a 7-fold increase in the primary outcome among women undergoing supraumbilical vertical incisions. We also investigated the following secondary outcomes: wound complications (a composite of superficial and deep surgical site infection, wound dehiscence, or return to the operating room to address the wound), need for transfusion, postoperative length of stay more than 3 days, total minutes of surgery, drain placement, endomyometritis, and estimated blood loss of more than $1000 \mathrm{~mL}$. Potential confounding variables included age, body mass index, preexisting maternal diseases, number of previous abdominal surgeries, use of surgical drains, use of preoperative antibiotics, and gestational age at delivery.

Data analysis was performed using SAS 9.3 (SAS Institute Inc., Cary, NC). Two-sample $t$-test and chi-squared or Fisher's exact test was used to compare continuous and categorical data, respectively. Multivariate logistic regression analysis was performed to account for confounding variables.

\section{Results}

During the study period, 45 women delivered infants through a supraumbilical vertical incision. Two women were excluded from the cohort group due to incomplete records. Patient demographics are listed in Table 1. Our cohort group was more likely to include older women with a higher body mass index and higher prevalence of diabetes who delivered at an earlier gestational age compared to controls. All patients received preoperative antibiotics. Placement of a surgical drain was based on the preference of the surgeon. There were no cases identified with a planned classical hysterotomy.

Classical uterine incision was more prevalent in women who had a supraumbilical skin incision compared to controls (Pfannenstiel incision) (OR, 24.6; 95\% CI, 9.0-66.8). Drain placement (OR, 6.5; 95\% CI, 2.6-16.2) and estimated blood loss greater than $1000 \mathrm{~mL}$ (OR, 3.4; 95\% CI, 1.4-8.4) occurred more frequently in women with supraumbilical incisions. Total operative time was longer among those who had a supraumbilical skin incision compared to controls (97 minutes versus 68 minutes, $P<.001)$. Outcomes are listed in Table 2.

After adjusting with multivariate analysis for confounders (age, body mass index, gestational age at delivery, diabetes, tobacco use, and use of a surgical drain), supraumbilical incision remained as an independent significant risk factor for undergoing classical hysterotomy, placement of a surgical drain, estimated blood loss more than $1000 \mathrm{~mL}$, and longer operative time (Table 3 ).

\section{Discussion}

Women with a body mass index greater than $40 \mathrm{~kg} / \mathrm{m}^{2}$ who have a supraumbilical vertical skin incision at the time of cesarean are more likely to undergo a classical hysterotomy than those who have a Pfannenstiel incision. Supraumbilical incisions are also associated with a greater risk of placement of a surgical drain, estimated blood loss more than $1000 \mathrm{~mL}$, and longer operative times when compared to Pfannenstiel skin incisions.

One of the strengths of this study is the number of patients derived from a single institution over a relatively short period of time; this may limit the possibility of different techniques and postoperative care practices. The limitations of our study are those inherent to retrospective designs. Our cohort subjects differed significantly from controls in age and prevalence of higher body mass index and diabetes; however, after adjusting for these and other confounders, our results showed that supraumbilical incision was an independent risk factor that led to classical hysterotomy incision. There is also potential of referral bias given that all the patients in this study 
TABLE 2: Outcomes.

\begin{tabular}{lcccc}
\hline Outcome & $\begin{array}{c}\text { Supraumbilical } \\
N=43\end{array}$ & $\begin{array}{c}\text { Pfannenstiel } \\
N=90\end{array}$ & OR & $95 \%$ CI \\
\hline Classical hysterotomy & $29(67 \%)$ & $7(8 \%)$ & 24.6 & $9.0-66.8$ \\
Wound complication & $8(19 \%)$ & $7(8 \%)$ & 2.7 & $.9-8.0$ \\
Blood transfusion & $7(16 \%)$ & $5(6 \%)$ & 3.3 & $.98-11.1$ \\
Length of stay $>3$ days & $33(77 \%)$ & $59(66 \%)$ & .0811 \\
Endomyometritis & $2(5 \%)$ & $2(2 \%)$ & .0557 \\
Drain placed & $18(42 \%)$ & $9(10 \%)$ & $.8-4.1$ & $.3-15.8$ \\
Estimated blood loss $>1$ liter & $14(33 \%)$ & $11(12 \%)$ & 6.5 & $2.6-16.2$ \\
Total minutes of surgery & $97 \pm 38$ & $68 \pm 30$ & 3.4 & $1.4-8.4$ \\
\hline
\end{tabular}

TABLE 3: Multivariate regression analysis.

\begin{tabular}{lccc}
\hline Outcome & OR & $95 \%$ CI & $P$ value \\
\hline Classical hysterotomy & 15.8 & $4.1,60.8$ & $<.0001$ \\
Drain & 7.8 & $2.3,26.7$ & .0010 \\
Estimated blood loss $>$ 1 liter & 6.4 & $2.0,20.1$ & .0022 \\
Total minutes of surgery & Slope $=27.5$ & & .0011 \\
\hline
\end{tabular}

Variables controlled for include age, body mass index, gestational age at delivery, and diabetes mellitus.

were from one regional perinatal center. The majority of the patients was of African American ancestry and had public insurance, which could limit the external validity of the study.

Our findings agree with those reported by other investigators. Alanis et. al. showed that women with body mass index greater than $40 \mathrm{~kg} / \mathrm{m}^{2}$ with vertical skin cesarean incisions were more likely to undergo classical hysterotomy; however, they did not differentiate between supraumbilical and infraumbilical vertical incisions [9].

The morbidity associated with classical cesarean delivery and women with a prior classical hysterotomy has been widely reported $[10,11]$. Obesity has been shown to be an independent risk factor for cesarean delivery [12]. As the prevalence of morbid obesity rises, obstetricians caring for these women during pregnancy will be faced with decisions regarding the best surgical approach should these patients require cesarean delivery. We believe that our results provide support for advocating Pfannenstiel skin incisions in women with body mass index greater than $40 \mathrm{~kg} / \mathrm{m}^{2}$ when access to the lower abdomen is feasible. Other investigators support this approach as well despite the traditional concept that intraoperative exposure is restricted whenever using Pfannenstiel incisions [9].

\section{Conclusions}

In conclusion, supraumbilical vertical approach in women with body mass index greater than $40 \mathrm{~kg} / \mathrm{m}^{2}$ undergoing cesarean section is associated with higher rate of classical hysterotomy, greater blood loss, and longer operative time when compared to Pfannenstiel incisions. The importance of these findings allows clinicians to properly counsel these women who undergo cesarean delivery.

\section{References}

[1] K. M. Flegal, M. D. Carroll, C. L. Ogden, and L. R. Curtin, "Prevalence and trends in obesity among US adults, 1999-2008," Journal of the American Medical Association, vol. 303, no. 3, pp. 235-241, 2010.

[2] ACOG Committee Opinion number 315, "Obesity in pregnancy," Obstetrics \& Gynecology, vol. 106, no. 3, pp. 671-675, 2005.

[3] S. Cnattingius, R. Bergström, L. Lipworth, and M. S. Kramer, "Prepregnancy weight and the risk of adverse pregnancy outcomes," New England Journal of Medicine, vol. 338, no. 3, pp. 147-152, 1998.

[4] M. I. Cedergren, "Maternal morbid obesity and the risk of adverse pregnancy outcome," Obstetrics \& Gynecology, vol. 103, no. 2, pp. 219-224, 2004.

[5] H. M. Salihu, A.-L. Dunlop, M. Hedayatzadeh, A. P. Alio, R. S. Kirby, and G. R. Alexander, "Extreme obesity and risk of stillbirth among black and white gravidas," Obstetrics \& Gynecology, vol. 110, no. 3, pp. 552-557, 2007.

[6] E. A. Nohr, B. H. Bech, M. J. Davies, M. Frydenberg, T. B. Henriken, and J. Olsen, "Prepregnancy obesity and fetal death: a study within the Danish National Birth Cohort," Obstetrics \& Gynecology, vol. 106, no. 2, pp. 250-259, 2005.

[7] D. Getahun, C. V. Ananth, Y. Oyelese, M. R. Chavez, R. S. Kirby, and J. C. Smulian, "Primary preeclampsia in the second pregnancy: effects of changes in prepregnancy body mass index between pregnancies," Obstetrics \& Gynecology, vol. 110, no. 6, pp. 1319-1325, 2007.

[8] J. A. Rock, H. W. Jones, and R. W. Te Linde, Te Linde's Operative Gynecology, Wolters Kluwer Health/Lippincott Williams \& Wilkins, Philadelphia, Pa, USA, 10th edition, 2008.

[9] M. C. Alanis, M. S. Villers, T. L. Law, E. M. Steadman, and C. J. Robinson, "Complications of cesarean delivery in the massively obese parturient," American Journal of Obstetrics and Gynecology, vol. 203, no. 3, pp. 271-277, 2010.

[10] L. S. Patterson, C. M. O’Connell, and T. F. Baskett, "Maternal and perinatal morbidity associated with classic and inverted $\mathrm{T}$ cesarean incisions," Obstetrics \& Gynecology, vol. 100, no. 4, pp. 633-637, 2002.

[11] T. Bakhshi, M. B. Landon, Y. Lai et al., "Maternal and neonatal outcomes of repeat cesarean delivery in women with a prior classical versus low transverse uterine incision," American Journal of Perinatology, vol. 27, no. 10, pp. 791-795, 2010.

[12] H. E. Robinson, C. M. O'Connell, K. S. Joseph, and N. L. McLeod, "Maternal outcomes in pregnancies complicated by obesity," Obstetrics \& Gynecology, vol. 106, no. 6, pp. 1357-1364, 2005. 


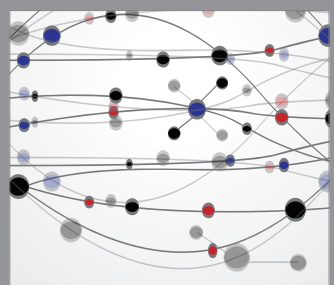

The Scientific World Journal
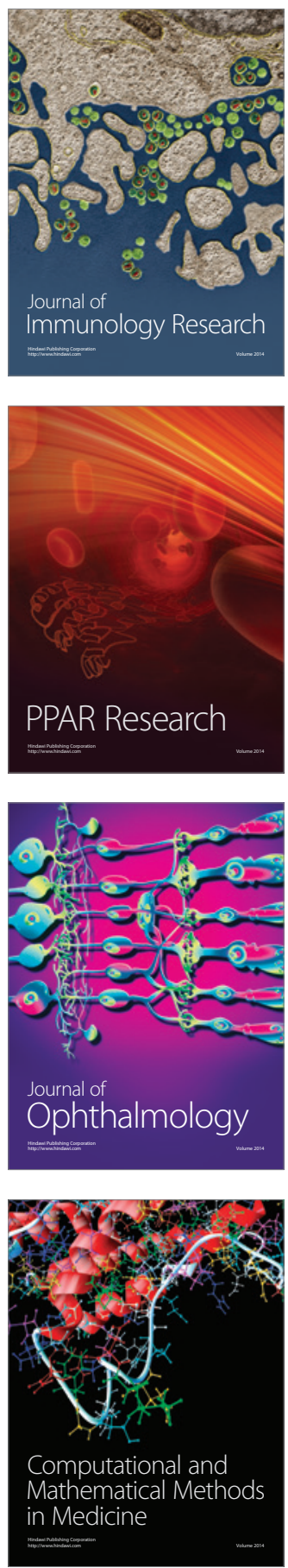

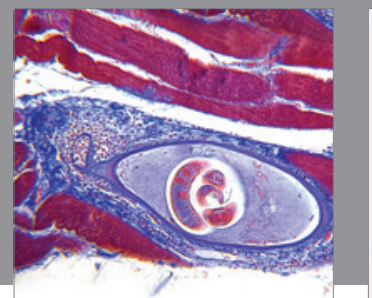

Gastroenterology

Research and Practice
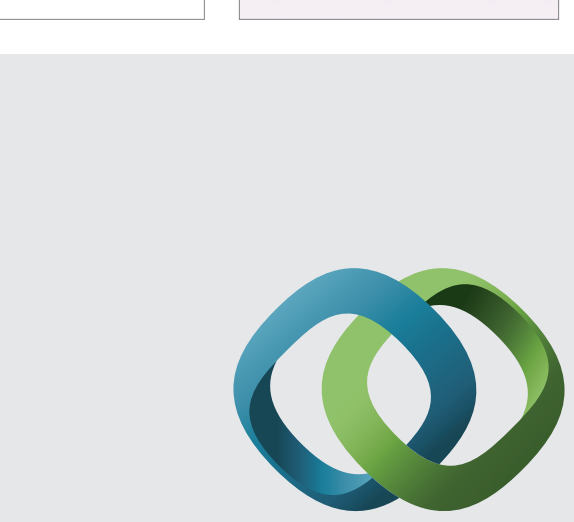

\section{Hindawi}

Submit your manuscripts at

http://www.hindawi.com
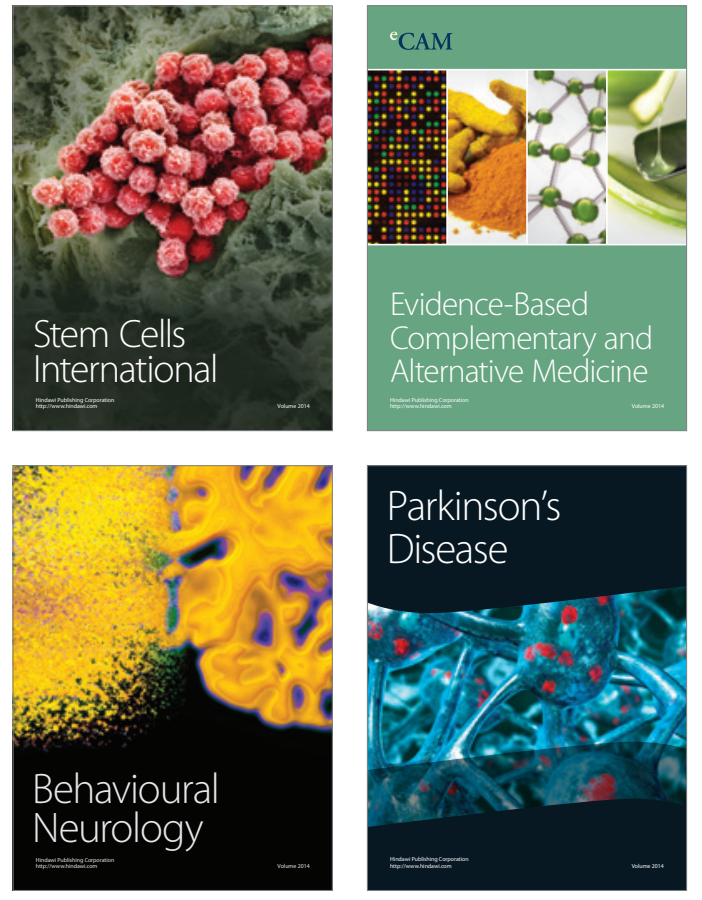
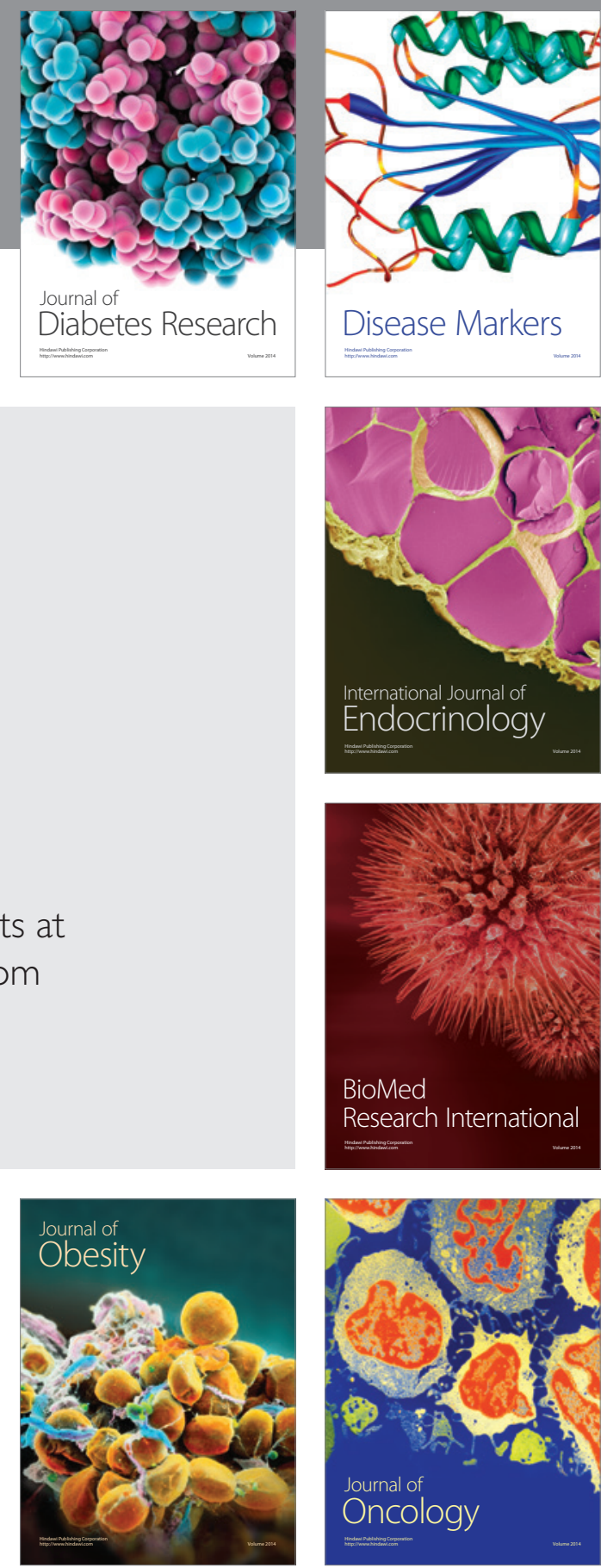

Disease Markers
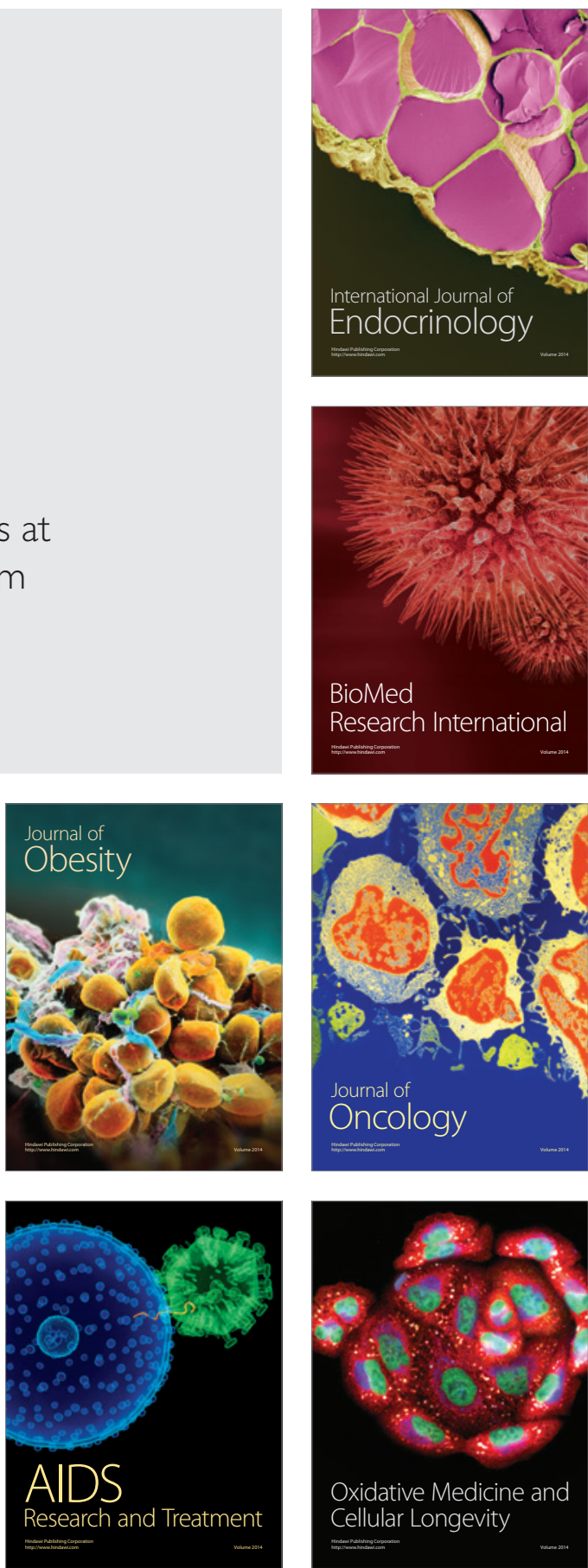